\title{
Rechnung für Basistarifversicherte
}

Das Wissen in den Praxen zum Basistarif ist gering. Es unterlaufen zunehmend Fehler. Verkalkulieren ist einer davon. (Siehe beispielsweise bei www.alex-za.de "Basistarif".)

\begin{tabular}{|c|c|c|c|c|}
\hline GOZ-Nr. & Leistung & Faktor & Anzahl & $€$-Betrag \\
\hline 0010 & eingehende Untersuchung & 2,0 & 1 & $11,25 €$ \\
\hline 1040 & professionelle Zahnreinigung & 2,0 & 24 & $75,59 €$ \\
\hline 2010 & Behandlung überempfindlicher Zahnflächen & 2,0 & 2 & $11,25 €$ \\
\hline 2310 & Wiederherstellung Verblendung an Prothese & 2,0 & 6 & $97,86 €$ \\
\hline 4060 & Kontrolle, Nachreinigung, Politur von Zähnen & 2,0 & 24 & $18,90 €$ \\
\hline \multirow[t]{2}{*}{4020} & Mundschleimhautbehandlung & 2,0 & 1 & $5,06 €$ \\
\hline & & & & $219,91 €$ \\
\hline
\end{tabular}

T2 Rechnung über das zahnärztliche Honorar nach dem BEMA mit aktuellem GKV-Punktwert

\begin{tabular}{lllll}
\hline BEMA-Nr. & Leistung & Faktor & Anzahl & $€$-Betrag \\
01 & eingehende Untersuchung & 1,0 & 1 & $18,48 €$ \\
107 & Zahnsteinentfernung je Sitzung & 1,0 & 1 & $16,42 €$ \\
10 & Behandlung überempfindlicher Zahnflächen & 1,0 & 1 & $6,16 €$ \\
$24 \mathrm{~b}$ & Wiederherstellung Verblendung an Prothese & 1,0 & 6 & $264,81 €$ \\
- & Kontrolle, Nachreinigung, Politur von Zähnen & - & - & - \\
105 & Mundschleimhautbehandlung & 1,0 & 1 & $8,21 €$ \\
\hline & & Differenzzu 2,0 fach GOZ: & $-94,17 €$ \\
\hline
\end{tabular}

Der Basistarif ist an die Leistungen der gesetzlichen Krankenversicherung angelehnt. Der Basistarif-Versicherte ist dennoch privat versichert. Dann ist die Rechnungslegung nach den Maßgaben der Gebührenordnung für Zahnärzte (GOZ) zutreffend, aber mit Ausnahmen: Die Berechnung der Gebührenhöhe für Leistungen der GOZ ist nur bis zum 2-fachen Satz möglich, wenn eine Behandlung zu Bedingungen des Basistarifs wirksam vereinbart wurde (in der Regel: GÖ̈ 1,8-fach, Rö 1,38-fach). Ein Beispiel ( Tab 1):

Zum durchschnittlichen Faktor 2,3-fach ergibt sich bereits eine Differenz von 32,99€.

\section{Höheres Honorar bei GKV-Versicherten}

Aktuell erhielte der Zahnarzt beim gesetzlich Versicherten in diesem Beispiel 94,17 Euro mehr Honorar als beim Versicherten im Basistarif bei Berechnung des 2,0-fachen GOZ-Satzes. Selbst bei vereinbarter Berechnung des 2,3-fachen Satzes wären es immerhin noch 61,19 Euro weniger als aktuell in der GKV für die ausreichende, zweckmäßige und wirtschaftliche Versorgung vergütet würde ( Tab 2).

Versicherten im Basistarif ist oft nicht bewusst, dass bei Zahnersatz oder dessen Reparatur Eigenanteile entstehen, wie dies auch bei gesetzlich Versicherten der Fall ist.

Von der Gebühr für die Wiederherstellung der Verblendung zum 2,0-fachen Satz werden zum Beispiel nur 65\% erstattet, wie von den „,anerkannten Material- und Laborkosten“ nach BEL II. Die Erstattung von 65 Prozent für Zahnersatz resultiert aus einem Vergleich mit der GKV.

\section{Fazit}

Der Basisversicherte ist ein Patient, der oft eigene Probleme hat. Er bereitet in der Praxis gelegentlich auch Probleme. Er benötigt einerseits Zuwendung, aber auch offene Ansprache. Daraus sollte eine schriftliche Vereinbarung der Gebührenhöhe gemäß $\$ 2(1,2)$ GOZ resultieren: individuell auf den Patienten zutreffend, selbst bei Sätzen bis zu 2,3-fach.

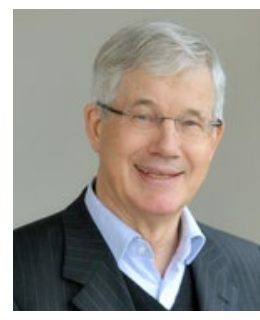

Dr. Peter H.G. Esser

GOZ-Experte und -Berater der ZA eG www.za-abrechnung.de 